\title{
Comparison of Bone Microarchitecture Between Adult Osteogenesis Imperfecta and Early-Onset Osteoporosis
}

\author{
Tim Rolvien ${ }^{1,2}$ (D) Julian Stürznickel ${ }^{1} \cdot$ Felix N. Schmidt ${ }^{1}$. Sebastian Butscheidt ${ }^{1} \cdot$ Tobias Schmidt $^{1,2} \cdot$ Björn Busse $^{1}$. \\ Stefan Mundlos ${ }^{3,4,5} \cdot$ Thorsten Schinke $^{1} \cdot$ Uwe Kornak $^{3,4,5} \cdot$ Michael Amling $^{1} \cdot$ Ralf Oheim $^{1}$
}

Received: 20 March 2018 / Accepted: 19 June 2018 / Published online: 26 June 2018

(c) Springer Science+Business Media, LLC, part of Springer Nature 2018

\begin{abstract}
Diagnosis and management of adult individuals with low bone mass and increased bone fragility before the age of 50 can be challenging. A number of these patients are diagnosed with mild osteogenesis imperfecta (OI) through detection of COL1AI or COL1A2 mutations; however, a clinical differentiation from early-onset osteoporosis (EOOP) may be difficult. The purpose of this study was to determine the bone microstructural differences between mild OI and EOOP patients. 29 patients showed mutations in COL1A1 or COL1A2 and were classified as OI. Skeletal assessment included dual-energy X-ray absorptiometry (DXA), high-resolution peripheral quantitative computed tomography (HR-pQCT), and bone turnover serum analyses. Bone microstructure of 21/29 OI patients was assessed and compared to 23 age- and sex-matched patients clinically classified EOOP but without mutations in the known disease genes as well as to 20 healthy controls. In the OI patients, we did not observe an age-dependent decrease in DXA Z-scores. HR-pQCT revealed a significant reduction in volumetric BMD and microstructural parameters in the distal radius and tibia in both the OI and EOOP cohorts compared to the healthy controls. When comparing the bone microstructure of OI patients with the EOOP cohort, significant differences were found in terms of bone geometry in the radius, while no significant changes were detected in all other HR-pQCT parameters at the radius and tibia. Taken together, adult mild OI patients demonstrate a predominantly high bone turnover trabecular bone loss syndrome that shows minor microstructural differences compared to EOOP without mutation detection.
\end{abstract}

Keywords Osteogenesis imperfecta $\cdot \mathrm{HR}-\mathrm{pQCT} \cdot$ Collagen type I Early-onset osteoporosis $\cdot$ Gene panel sequencing

Tim Rolvien and Julian Stürznickel have contributed equally.

Electronic supplementary material The online version of this article (https://doi.org/10.1007/s00223-018-0447-8) contains supplementary material, which is available to authorized users.

Tim Rolvien

t.rolvien@uke.de

1 Department of Osteology and Biomechanics, University Medical Center Hamburg-Eppendorf, Lottestr. 59, 22529 Hamburg, Germany

2 Department of Orthopedics, University Medical Center Hamburg-Eppendorf, Martinistraße 52, 20246 Hamburg, Germany

3 Institute of Medical Genetics and Human Genetics, Charité Universitätsmedizin Berlin, 13353 Berlin, Germany

4 Berlin-Brandenburg School for Regenerative Therapies, Charité-Universitätsmedizin Berlin, Berlin, Germany

5 FG Development and Disease, Max Planck Institute for Molecular Genetics, Berlin, Germany

\section{Introduction}

Although fracture risk typically increases with age in adults, individuals with recurrent fractures and low BMD can be also found among younger adults. An important differential diagnosis for these individuals is mild osteogenesis imperfecta (OI). OI is a rare genetic bone disorder that is mainly caused by autosomal dominant mutations in the collagen, type I, alpha 1 (COL1A1) or collagen, type I, alpha 2 (COL1A2) gene [1]. While the clinical picture of OI varies, the most common features are short stature and fractures after no or low trauma. Clinically, four major types I-IV are described ranging from mild to lethal forms, which usually manifest in childhood [2,3]. Overlapping with the typical forms of OI being caused by collagen I mutations, earlyonset osteoporosis (EOOP) is a condition involving low bone mass and the occurrence of fragility fractures before the age of 50 years. EOOP may be caused by single-gene mutations (i.e., WNT1 [4, 5], LRP5 [6], PLS3 [7]), but in many cases 
no monogenetic bone disorders may be detected. In fact, it might be challenging to differentiate mild or late-onset $\mathrm{OI}$ from EOOP, especially when dealing with adult patients and no other symptoms like blue sclerae or teeth abnormalities are present. The advent of modern techniques such as nextgeneration sequencing (NGS) allows parallel screening of many potential disease genes including collagen I mutations; thus making the need of a clear clinical diagnosis prior to genetic testing obsolete.

The first study that had analyzed the skeletal status of adult OI patients beyond dual-energy X-ray absorptiometry (DXA) has reported an affected cortical thickness and reduced volumetric bone density in quantitative computed tomography (QCT) [8]. High-resolution peripheral quantitative computed tomography (HR-pQCT) is a non-invasive technique that allows a separate quantification of trabecular and cortical volumetric BMD (Tb.BMD, Ct.BMD) as well as bone microstructure and geometry of the peripheral bone in the distal radius and tibia [9]. The microstructural alterations in bone structure have been found to correlate with fracture risk in postmenopausal women [10]. In a previous HR-pQCT study on bone geometry, density, and microarchitecture in OI type I patients, areal and volumetric BMD, bone area, and trabecular number were lower in OI type I compared to healthy age- and sex-matched controls [11]. Furthermore, the alterations in bone mass and architecture have been found to differ between the clinical forms of OI type I, III, and IV [12] and to correlate with collagen structure, based on quantitative and qualitative collagen analyses from fibroblasts and serum [13]. In general a genotype-phenotype correlation between the type I collagen loci (i.e., COL1A1 or COL1A2) has been addressed previously [14, 15].

It is currently unknown if adult patients with OI due to COL1A1 or COL1A2 mutations differ in bone microstructure compared to patients with EOOP without mutations in known disease genes. In this study, we demonstrate the skeletal and genetic status of a German cohort of 29 adult OI patients harboring both known and novel COL1A1 and COL1A2 mutations, while we outline the microstructural bone alterations of 21 of these OI patients compared to 23 patients with EOOP without mutations in 386 known disease genes as well as to 20 healthy controls.

\section{Materials and Methods}

\section{Patients}

This OI study group consisted of 29 patients with COL1A1 or COL1A2 mutations, which were diagnosed with OI (type I or IV). In fact, all adult OI type I or IV patients from our outpatient clinic were included in this study, whereas patients with OI type III were not included. HR-pQCT was performed in 21 of these 29 OI patients. The EOOP group consisted of 23 patients with no relevant mutations in 386 disease genes explaining the respective phenotypes. This group of patients was similar in age, sex ratio, and DXA results compared to the OI group and represents all sex- and age-matched mutation negative patients from a database of patients with EOOP and other monogenetic bone disorders in the setting of a specialized osteologic outpatient clinic (National Bone Board). Patients with detected gene mutations in the 386 genes with known impact on bone status, among others WNT1, LRP5, PLS 3, were excluded from both groups. Furthermore, patients with peculiar forms of OI (i.e., high bone mass OI through collagen I cleavage site mutations [16]) were excluded from the OI group. Other exclusion criteria were glucocorticoid treatment, cancer, other metabolic bone diseases, renal dysfunction (GFR $<60 \mathrm{ml} /$ $\min$ ), and severe underweight at the time of the study or any time in the past.

Data were compared with 20 healthy, age-, and sexmatched controls. These patients were screened for bone status due to an upcoming spine surgery (e.g., disc herniation) and risk evaluation of post-operative screw loosening. This study was performed in accordance with the local ethics committee (PV5364) and the Declaration of Helsinki. An overview of the study groups is shown in Tables 1 and 2.

\section{Genetic Analysis}

To test for disease-causing genetic variants, DNA was isolated from the patients' peripheral blood, and 103 exons and flanking introns of COL1A1 and COL1A2 were amplified by PCR, followed by Sanger sequencing on an ABI 3730 sequencer (Thermo Scientific). In case no type I collagen mutation was identified, all of them proceeded to a customdesigned SureSelect XT gene panel (Agilent, Santa Clara, CA, USA) comprising 386 genes in which mutations have been associated with changes in bone mass, skeletal dysplasias, dysostoses, or connective tissue diseases [skeletal disorder-associated genome (sDAG) gene panel] as described recently [17]. The pathogenicity of the prioritized variants was judged using PhenIX [18], which integrates variant scoring by PolyPhen, SIFT, and MutationTaster.

\section{Assessment of Skeletal Status}

The areal bone mineral density (aBMD) was evaluated in all patients using dual-energy X-ray absorptiometry at the hip and spine (DXA, Lunar iDXA, GE Healthcare; Madison, WI, USA). Biochemical bone turnover markers were analyzed from all OI patients' serum (osteocalcin, bone-specific alkaline phosphatase) and urine (DPD cross links) at initial presentation. Fracture history and current fracture status was determined for all patients by comprehensive assessment of 
Table 1 Overview of the 29 OI patients including sex, age, mutation sequence, novelty, and clinical features

\begin{tabular}{|c|c|c|c|c|c|c|c|c|c|c|}
\hline No. & Sex & Age & Gene & Mutation & Novel & $\begin{array}{l}\text { Vertebral } \\
\text { fractures }\end{array}$ & $\begin{array}{l}\text { Extravertebral } \\
\text { fractures }\end{array}$ & $\begin{array}{l}\text { Childhood } \\
\text { fractures }\end{array}$ & Blue sclera & HR-pQCT \\
\hline 1 & $\mathrm{~m}$ & 80 & COL1A1 & p.Asp1065Glu & Yes & 4 & $>4$ & No & No & Yes \\
\hline 2 & $\mathrm{f}$ & 51 & COL1A1 & p.Asp1065Glu & Yes & 0 & 2 & No & Yes & Yes \\
\hline 3 & $\mathrm{~m}$ & 40 & COL1A1 & p.Asp1065Glu & Yes & 0 & 4 & No & No & Yes \\
\hline 4 & $\mathrm{f}$ & 71 & COL1A1 & p.Pro951fs & No & 0 & 6 & Yes & Yes & No \\
\hline 5 & $\mathrm{~m}$ & 23 & COL1A1 & p.Pro951fs & No & 0 & 6 & Yes & No & Yes \\
\hline 6 & $\mathrm{f}$ & 50 & COL1A1 & p.Pro951fs & No & 0 & 5 & Yes & No & Yes \\
\hline 7 & $\mathrm{f}$ & 42 & COL1A1 & c.1054_1056del+2del & No & 9 & 1 & No & No & Yes \\
\hline 8 & $\mathrm{f}$ & 74 & COL1A1 & p.Gln199fs & No & 2 & 0 & No & Yes & Yes \\
\hline 9 & $\mathrm{~m}$ & 41 & COL1A1 & p.Asn1254fs & Yes & 0 & 8 & No & No & Yes \\
\hline 10 & $\mathrm{~m}$ & 51 & COL1A1 & p.Gly1178fs & Yes & 0 & 0 & No & No & Yes \\
\hline 11 & $\mathrm{f}$ & 68 & COL1A1 & p.Gly233Arg & Yes & 1 & 0 & No & No & Yes \\
\hline 12 & $\mathrm{f}$ & 46 & COL1A1 & p.Pro871fs & Yes & 0 & 1 & No & No & No \\
\hline 13 & $\mathrm{f}$ & 35 & COL1A1 & p.Pro871fs & Yes & 0 & $>20$ & Yes & Yes & No \\
\hline 14 & $\mathrm{~m}$ & 19 & COL1A1 & p.Gly194Valfs & No & 0 & 3 & Yes & No & No \\
\hline 15 & $\mathrm{f}$ & 29 & COL1A1 & c. $1614+1 \mathrm{G}>\mathrm{A}$ & No & 6 & 8 & No & No & Yes \\
\hline 16 & $\mathrm{f}$ & 57 & COL1A1 & p.Lys538Argfs & Yes & 4 & $>10$ & Yes & No & No \\
\hline 17 & $\mathrm{~m}$ & 61 & COL1A1 & c. $2830-1 \mathrm{G}>\mathrm{A}$ & No & 0 & 3 & No & No & No \\
\hline 18 & $\mathrm{~m}$ & 31 & COL1A1 & p.Tyr1216Thrfs & Yes & 0 & $>20$ & No & Yes & Yes \\
\hline 19 & $\mathrm{f}$ & 61 & COL1A1 & p.Tyr1216Thrfs & Yes & 2 & 3 & No & No & Yes \\
\hline 20 & $\mathrm{~m}$ & 41 & COL1A1 & p.Pro205Ala & No & 4 & $>10$ & Yes & No & Yes \\
\hline 21 & $\mathrm{~m}$ & 33 & COL1A1 & p.Gly692Valfs*74 & No & 5 & 5 & Yes & No & Yes \\
\hline 22 & $\mathrm{~m}$ & 28 & COL1A2 & p.Gly193Ser & No & 0 & 12 & Yes & No & Yes \\
\hline 23 & $\mathrm{f}$ & 31 & COL1A2 & p.Gly292Ser & No & 6 & 1 & No & No & Yes \\
\hline 24 & $\mathrm{f}$ & 58 & COL1A2 & p.Gly292Ser & No & 0 & 1 & No & No & No \\
\hline 25 & $\mathrm{f}$ & 52 & COL1A2 & p.Gly772Ser & No & 0 & 6 & Yes & No & Yes \\
\hline 26 & $\mathrm{~m}$ & 37 & COL1A2 & p.Lys1288Argfs & Yes & 6 & $>35$ & Yes & Yes & Yes \\
\hline 27 & $\mathrm{~m}$ & 49 & COL1A2 & p.Gly292Ser & No & 2 & 3 & No & No & No \\
\hline 28 & $\mathrm{f}$ & 59 & COL1A2 & p.Gly724Glu & Yes & 4 & 0 & No & No & Yes \\
\hline 29 & $\mathrm{~m}$ & 49 & COL1A2 & p.Gly1105Ser & No & 0 & 5 & No & No & Yes \\
\hline
\end{tabular}

medical history as well as radiography or vertebral morphometry by DXA.

Bone microstructure was further analyzed using high-resolution peripheral quantitative computed tomography (HRpQCT; XtremeCT, Scanco Medical, Switzerland) at the nondominant distal radius and tibia in a standardized procedure as previously described by our group and using the in vivo protocol [19-21]. HR-pQCT could only be performed in $21 / 29$ OI patients due to i.e., deformities or short extremities. Bone geometric parameters included cortical bone area $\left(\mathrm{Ct}\right.$. bone area, $\mathrm{mm}^{2}$ ) and trabecular bone area ( $\mathrm{Tb}$. bone area, $\mathrm{mm}^{2}$ ) and volumetric bone mineral density (vBMD) measurements included total vBMD (Total BMD, mgHA/ $\mathrm{cm}^{3}$ ), cortical BMD (Ct.BMD, $\left.\mathrm{mgHA} / \mathrm{cm}^{3}\right)$, and trabecular BMD (Tb.BMD, mgHA/ $\left./ \mathrm{cm}^{3}\right)$. Bone microstructure values are similar to those used in bone histology and include bone volume-to-total volume ratio (BT/TV), trabecular number (Tb.N, 1/mm), separation (Tb.Sp, mm), and thickness (Tb.
Th, mm), as well as cortical thickness (Ct.Th, mm) and cortical perimeter (Ct.Pm, mm).

\section{Statistical Analysis}

Statistical analysis was performed using GraphPad Prism version 7.0 software (GraphPad Software, Inc., USA). Normal distribution of the data was tested with the Kolmogorov-Smirnov test. To test for significant changes in bone microstructural parameters between the groups, ANOVA was performed. Data are presented as mean \pm standard error of mean (SEM) and in boxplots to show the variability within the sample (including the median, the two quartiles and the two extremes). $p$ Values of $<0.05$ were considered statistically significant. Age-dependent changes in DXA $Z$-scores were tested using linear regression analysis (Pearson correlation). 
Table 2 Patient characteristics of the three study groups including subdivision of the OI cohort in different mutation types

\begin{tabular}{|c|c|c|c|c|c|c|}
\hline Parameter & OI & OI—glycine mutation & OI-frameshift mutation & OI—other mutation & EOOP & Control \\
\hline $\begin{array}{l}\text { Number of } \\
\text { patients (w/ } \\
\text { HR-pQCT) }\end{array}$ & $29(21)$ & $8(6)$ & $14(9)$ & $7(6)$ & 23 & 20 \\
\hline $\begin{array}{l}\text { Age, year } \pm \text { SD } \\
(\text { w/HR-pQCT) }\end{array}$ & $\begin{array}{l}47.1 \pm 15.6 \\
\quad(46.2 \pm 15.6)\end{array}$ & $49.3 \pm 13.7$ & $44.9 \pm 16.8$ & $49.1 \pm 16.9$ & $46.04 \pm 10.1$ & $48.8 \pm 16.3$ \\
\hline Gender, $\mathrm{m} / \mathrm{f}$ & $14 / 15$ & $3 / 5$ & $7 / 7$ & $4 / 3$ & $13 / 10$ & $9 / 11$ \\
\hline $\begin{array}{l}\text { Body height } \\
\mathrm{m} / \mathrm{f}, \mathrm{cm} \pm \mathrm{SD}\end{array}$ & $\begin{array}{l}176.2 \pm 5.8 \\
\quad / 159.2 \pm 5.5\end{array}$ & $181.3 \pm 5.3 / 161.8 \pm 5.8$ & $176 \pm 4.9 / 156.6 \pm 5.7$ & $172.8 \pm 6.2 / 160.3 \pm 3.1$ & $178.9 \pm 10.1 / 167.9 \pm 9.5$ & $177 \pm 7.1 / 169 \pm 6.3$ \\
\hline $\begin{array}{l}\text { Multiple } \\
\text { fractures in } \\
\text { childhood, } n\end{array}$ & 10 & 2 & 7 & 1 & 1 & $\mathrm{n} / \mathrm{a}$ \\
\hline $\begin{array}{l}\text { Vertebral frac- } \\
\text { tures, } n\end{array}$ & 14 & 4 & 5 & 4 & 8 & 0 \\
\hline $\begin{array}{l}\text { Other fractures, } \\
n\end{array}$ & 25 & 6 & 12 & 7 & 13 & 2 \\
\hline No fractures, $n$ & 1 & 0 & 1 & 0 & 1 & 18 \\
\hline $\begin{array}{c}\text { T-score (spinal) } \\
\text { mean } \pm \text { SD }\end{array}$ & $-2.58 \pm 1.3$ & $-3.2 \pm 1.5$ & $-2.1 \pm 1.2$ & $-3.1 \pm 1.0$ & $-2.9 \pm 0.9$ & $-0.2 \pm 1.3$ \\
\hline $\begin{array}{c}\text { T-score (hip) } \\
\text { mean } \pm \text { SD }\end{array}$ & $-2.0 \pm 1.4$ & $-1.5 \pm 1.2$ & $-1.9 \pm 1.6$ & $-2.6 \pm 0.9$ & $-2.1 \pm 0.8$ & $-0.2 \pm 1.4$ \\
\hline $\begin{array}{l}Z \text {-score (spinal) } \\
\text { mean } \pm \text { SD }\end{array}$ & $-2.2 \pm 1.2$ & $-2 \pm 1.4$ & $-2.1 \pm 1.1$ & $-1.8 \pm 2.5$ & $-2.4 \pm 1.1$ & $0.3 \pm 1.4$ \\
\hline $\begin{array}{c}Z \text {-score (hip) } \\
\text { mean } \pm \text { SD }\end{array}$ & $-1.6 \pm 1.3$ & $-1.3 \pm 1.1$ & $-1.5 \pm 1.3$ & $-1.5 \pm 1.9$ & $-1.5 \pm 1.2$ & $0.2 \pm 1.3$ \\
\hline $\begin{array}{l}\text { Bone-specific } \\
\text { therapy, } \%\end{array}$ & 51.7 & 62.5 & 57.1 & 28.6 & 43.5 & 0 \\
\hline
\end{tabular}

\section{Results}

\section{OI Patient Characteristics}

Within the group of adult OI patients, 10 patients (34.5\%) suffered from multiple fractures in early childhood, while 28 patients $(96.6 \%)$ suffered from at least one fracture until the current presentation. 6 patients $(20.7 \%)$ demonstrated with blue sclerae. From the patients with multiple fractures in childhood, some had been clinically diagnosed with OI during childhood. However, most patients were diagnosed with OI as adults. 15 patients $(51.7 \%)$ had received a bone-specific therapy throughout their lives. At present, 10 patients $(34.5 \%)$ received a bone-specific therapy including oral and intravenous bisphosphonates ( 8 patients) and denosumab (2 patients). Low vitamin D levels (25-OH$\mathrm{D} 3<30 \mu \mathrm{g} / \mathrm{l})$ were detected in 16 patients at initial presentation; and a daily vitamin D supplementation was prescribed to all. Mean body height was $176.2 \pm 5.8 \mathrm{~cm}$ (45th percentile) for male and $159.2 \pm 5.5 \mathrm{~cm}$ (25th percentile) for female patients. The clinical picture in our patients was compatible with OI type I or IV. Besides the presented clinical data (e.g., fracture status, blue sclerae, Table 1), no further clinical information was available.

\section{Mutation Spectrum}

20 different mutations were found in the 29 patients. COL1AI mutations were found in 21 patients, while in the remaining 8 patients COL1A2 mutations were detected. Nine of these mutations were novel, of which two were classical qualitative missense mutations affecting glycine or proline residues within the triple-helical domain. Six mutations had quantitative effects through frameshifts. In contrast, one mutation was an atypical missense mutation, albeit affecting highly conserved residues and absent or at least of extremely low frequency in the general population. Overall 14 patients carried either splice site or frameshift mutations. The quantitative effect of these mutations is usually associated with mild phenotypes. A comprehensive summary of all mutations is given in Table 1 . The OI patients were subdivided in three groups (a) with glycine mutations, (b) with frameshift mutation, and (c) with atypical missense (other) mutation (Table 2).

\section{Bone Density and Bone Turnover Markers of OI Patients}

According to DXA measurements, 25/29 patients had a $Z$-score $<-1.0$ at the lumbar spine or hip, while $17 / 29$ 
patients $(58.6 \%)$ presented with a $Z$-score $<-2.0$. Importantly, no age-dependent changes in DXA $Z$-score could be determined $\left(R^{2}=0.05, p=0.77\right.$, Fig. 1a, b). Bone turnover analysis revealed a moderate-to-high bone turnover, while bone formation markers were within normal range in most patients and bone resorption parameters were elevated in 15 patients (Supp. Fig. 1A-C). From the remaining 14 patients with normal bone resorption markers, 8 patients were currently on anti-resorptive treatments. Two patients with elevated levels of BAP were identified to have a very low vitamin D status and moderate secondary hyperparathyroidism, which was balanced within the first 3 months after the initiation of the vitamin D supplementation.

\section{Differences in Bone Microstructure Regarding OI Mutation Type and Between Adult OI and EOOP Patients Compared to Healthy Controls}

In both cohorts (OI and EOOP), the alterations in bone microstructure were highly variable compared to healthy controls ranging from severely impaired trabecular bone to almost normal trabecular and diminished cortical parameters or moderate combined trabecular and cortical bone loss (Fig. 2a-d). In the OI cohort, the subdivision in three groups based on mutation types did not reveal major significant differences regarding bone microstructure (Supp. Tables 1,2).

When comparing the OI patients with healthy controls in the radius, geometrical analysis revealed a significantly reduced cortical bone area $(p=0.033)$ but no changes with respect to trabecular area (Table 3 ). In the microstructural analysis, we found a significant reduction in BV/TV $(p<0.001)$, Tb.N $(p<0.001)$, and Tb.Sp $(p=0.011)$, but not in trabecular thickness (Tb.Th) or cortical thickness (Ct.Th) (Fig. 3; Table 3). Trabecular volumetric BMD but not cortical BMD was also significantly reduced in the distal radius of OI patients ( $p<0.001$; Fig. 3 ; Table 3$)$. In the distal tibia, Ct. bone area $(p=0.01), \mathrm{BV} / \mathrm{TV}, \mathrm{Tb} . \mathrm{N}$ $(p<0.001)$, Tb.Th $(p=0.046)$, Tb.Sp $(p=0.003)$, and Ct.Th $(p=0.018)$ were significantly reduced (Fig. 4; Table 4). Similar to the radius data, trabecular volumetric
Fig. 1 Bone mineral density expressed by DXA $Z$-scores in adult $\mathrm{OI}$ patients. a $Z$-scores in the lumbar spine (LS). b $Z$-scores in the hip. No agedependent decrease in DXA $Z$-score was observed
A

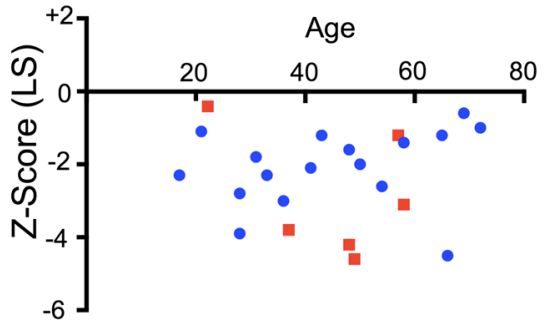

B

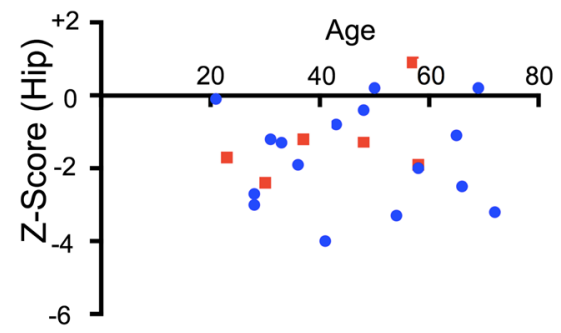

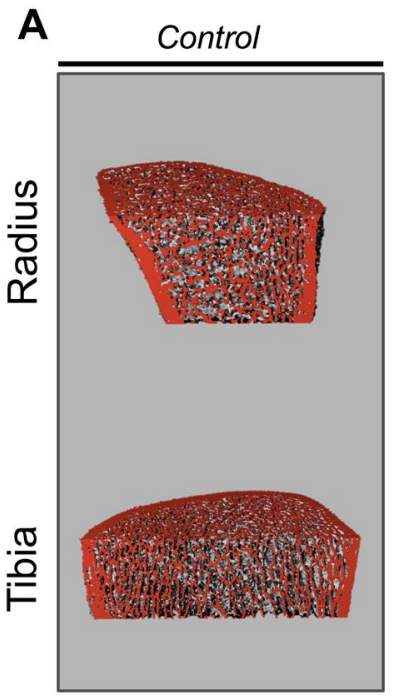

B
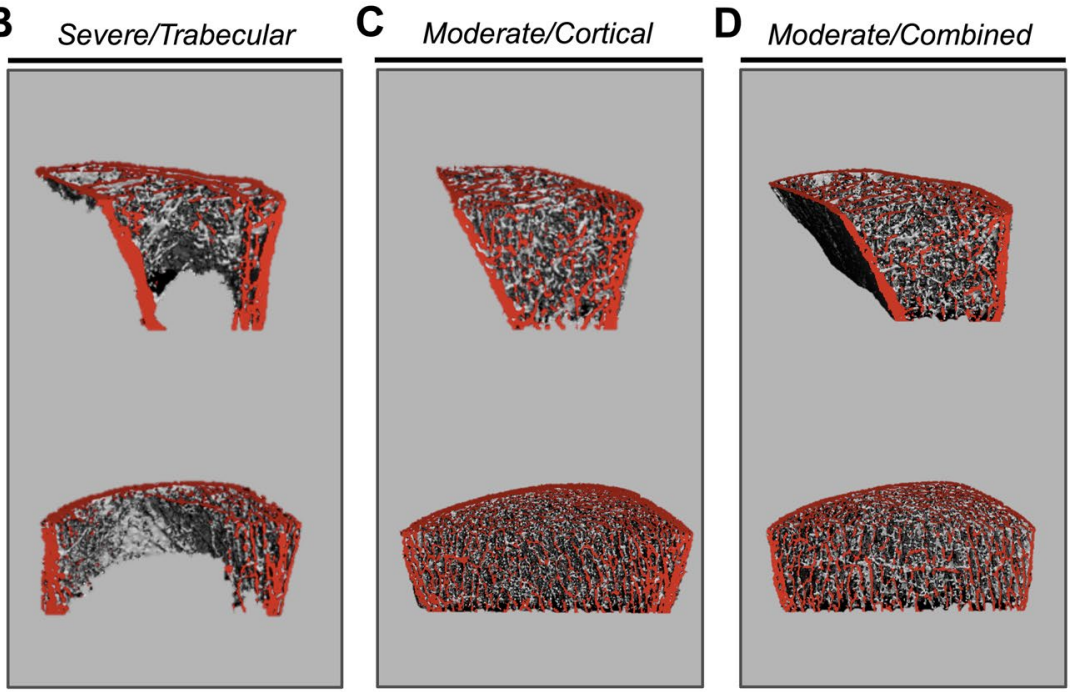

Fig. 2 High-resolution peripheral quantitative CT scans demonstrate the heterogeneity of microstructural alterations in OI and EOOP. a Healthy control. b Severely diminished trabecular microarchitecture in an OI patient. c Cortical bone loss but almost normal trabecular bone (OI patient). d Moderate, combined trabecular, and cortical bone loss (EOOP patient). Note that all three bone loss patterns $(\mathbf{b}-\mathbf{c})$ occurred in both groups 
Table 3 Bone microstructure assessed by HR-pQCT (radius)

OI $(n=21) \quad$ EOOP $(n=23) \quad$ Control $(n=20) \quad p$ Value $\quad p$ Value $\quad p$ Value

$\begin{array}{lll}\text { OI vs. EOOP } & \text { OI vs. control } & \text { EOOP vs. control }\end{array}$

Bone geometry

Ct. bone area $\left(\mathrm{mm}^{2}\right)$

Tb. bone area $\left(\mathrm{mm}^{2}\right)$

$51.89 \pm 2.74$

$69.13 \pm 7.21$

$366.4 \pm 41.87$

$61.77 \pm 3.56$

$264.5 \pm 13.91$

Cortical and trabecular microstructure

\begin{tabular}{|c|c|c|c|c|c|c|}
\hline Ct.Th $(\mathrm{mm})$ & $0.684 \pm 0.034$ & $0.707 \pm 0.044$ & $0.784 \pm 0.039$ & 0.691 & 0.061 & 0.206 \\
\hline Ct.Pm (mm) & $76.6 \pm 2.71$ & $89.48 \pm 3.36$ & $78.63 \pm 1.94$ & 0.007 & 0.551 & 0.014 \\
\hline $\mathrm{BV} / \mathrm{TV}$ (ratio) & $0.106 \pm 0.007$ & $0.108 \pm 0.008$ & $0.158 \pm 0.008$ & 0.913 & $<0.0001$ & $<0.0001$ \\
\hline Tb.N (1/mm) & $1.636 \pm 0.086$ & $1.672 \pm 0.087$ & $2.233 \pm 0.066$ & 0.774 & $<0.0001$ & $<0.0001$ \\
\hline Tb.Th (mm) & $0.066 \pm 0.003$ & $0.063 \pm 0.002$ & $0.071 \pm 0.003$ & 0.375 & 0.248 & 0.034 \\
\hline Tb.Sp (mm) & $0.624 \pm 0.085$ & $0.563 \pm 0.056$ & $0.385 \pm 0.015$ & 0.552 & 0.011 & 0.007 \\
\hline \multicolumn{7}{|c|}{ Volumetric bone mineral density } \\
\hline Total BMD (mg HA/ $\left.\mathrm{cm}^{3}\right)$ & $437.6 \pm 150.8$ & $276.6 \pm 13.26$ & $332.9 \pm 12.65$ & 0.272 & 0.504 & 0.004 \\
\hline Ct.BMD (mg HA/ $\left.\mathrm{cm}^{3}\right)$ & $813.8 \pm 12.64$ & $807.7 \pm 15.22$ & $818.8 \pm 9.363$ & 0.761 & 0.753 & 0.55 \\
\hline Tb.BMD (mg HA/ $\left./ \mathrm{cm}^{3}\right)$ & $127.4 \pm 7.93$ & $129 \pm 9.58$ & $190 \pm 9.97$ & 0.9 & $<0.0001$ & $<0.0001$ \\
\hline
\end{tabular}

Bold indicates significant differences $(p<0.05)$

Data are presented as mean \pm SEM

Fig. 3 Differences in bone microarchitecture in the distal radius of OI patients compared to patients with early-onset osteoporosis (EOOP) and healthy controls. Differences in a BV/TV, bone volume per tissue volume; b Tb.N, trabecular number; $\mathbf{c} \mathrm{Tb} . \mathrm{Th}$, trabecular thickness; d Ct.Th, cortical thickness; e Tb.BMD, trabecular bone mineral density; f Ct.BMD, cortical bone mineral density; ${ }^{*} p<0.05$. Data are presented in boxplots
A
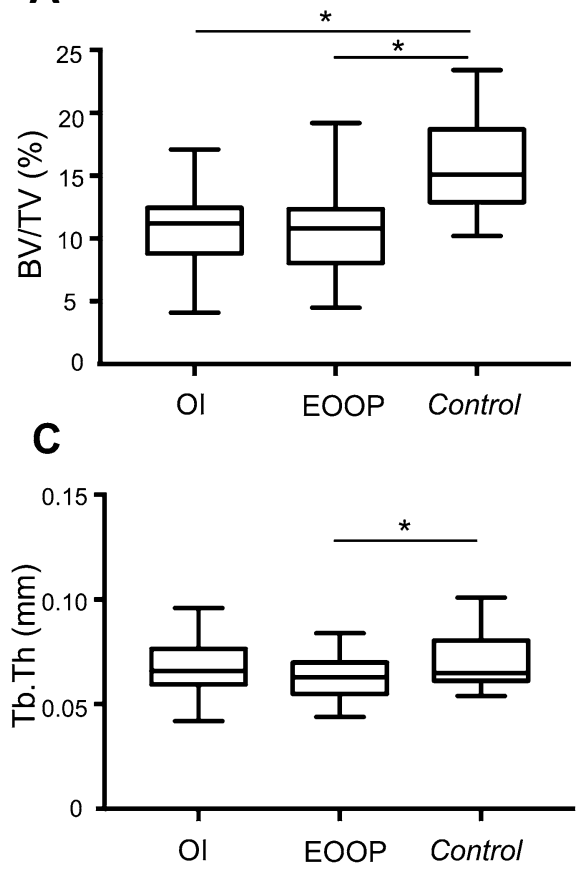

E

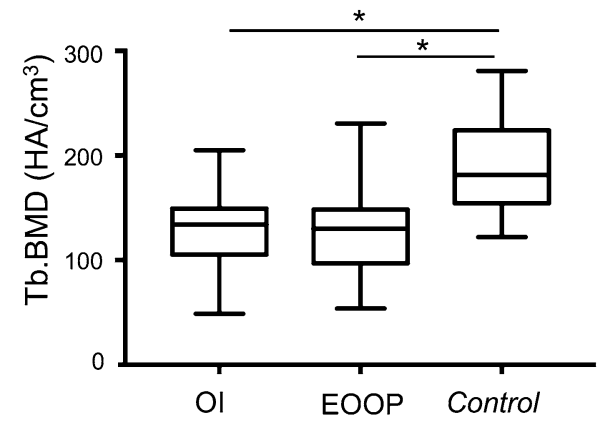

B
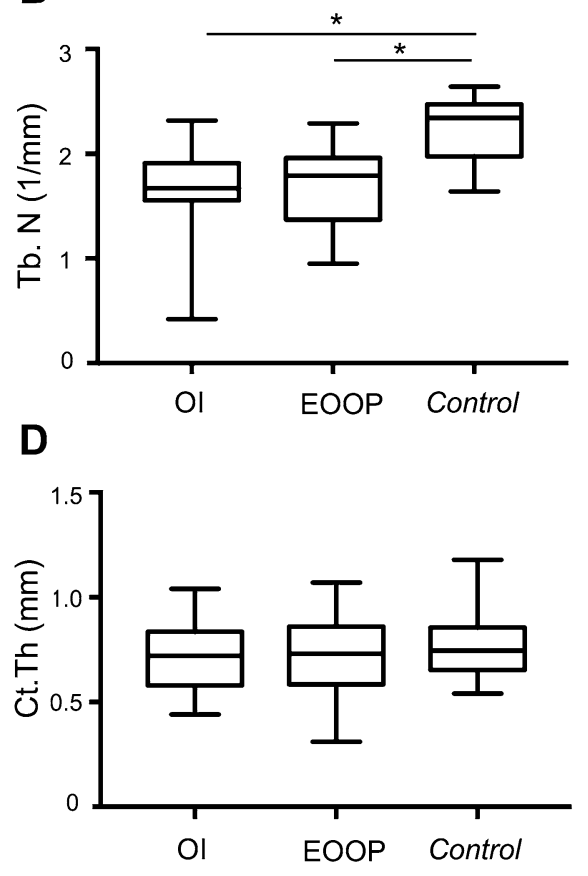

F

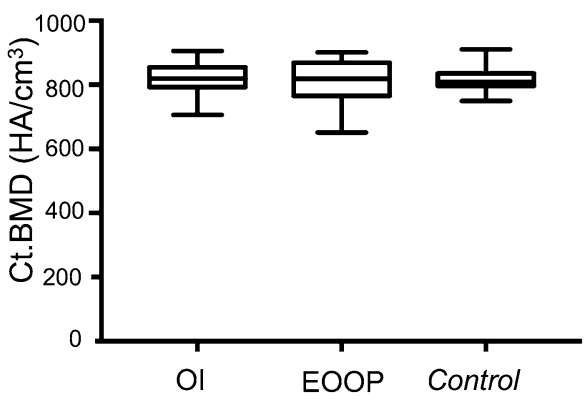


Fig. 4 Differences in bone microarchitecture in the distal tibia of OI patients compared to patients with early-onset osteoporosis (EOOP) and healthy controls. Differences in a $\mathrm{BV} / \mathrm{TV}$, bone volume per tissue volume; $\mathbf{b} \mathrm{Tb} . \mathrm{N}$, trabecular number; c Tb.Th, trabecular thickness; $\mathbf{d}$ Ct.Th, cortical thickness; e Tb.BMD, trabecular bone mineral density; $\mathbf{f}$ Ct.BMD, cortical bone mineral density; ${ }^{*} p<0.05$
A
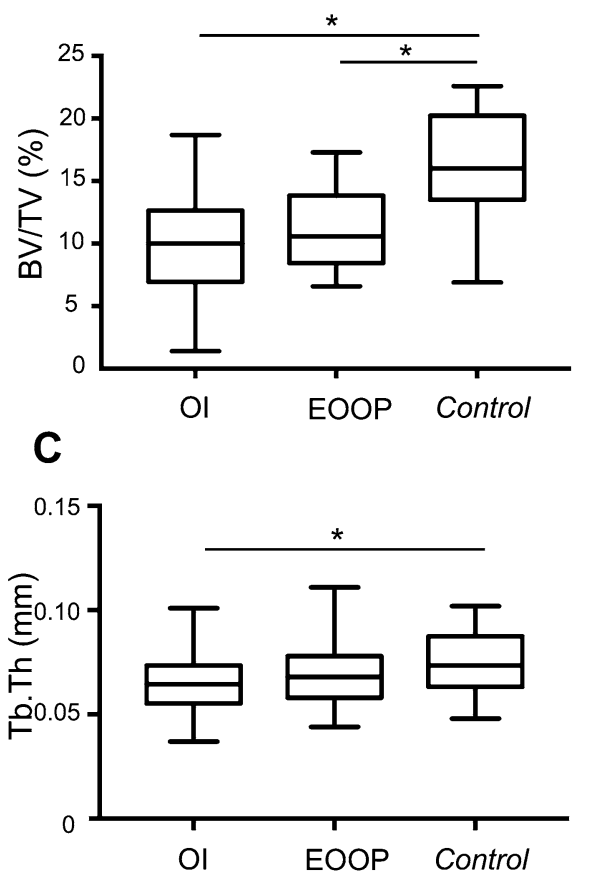

E

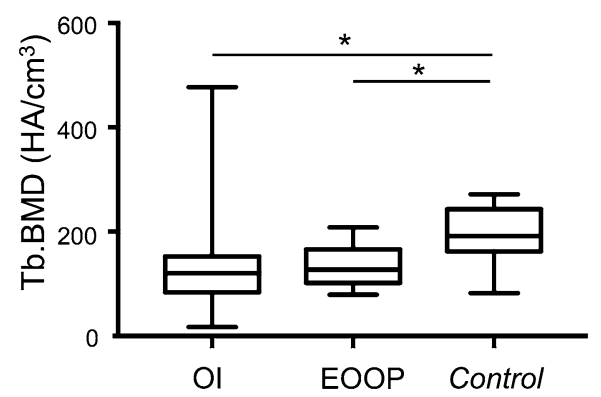

B

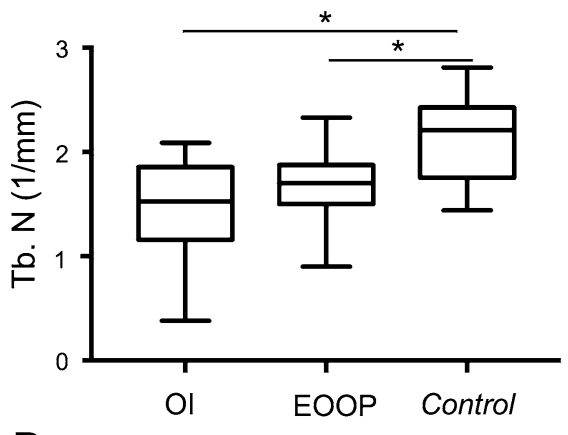

D

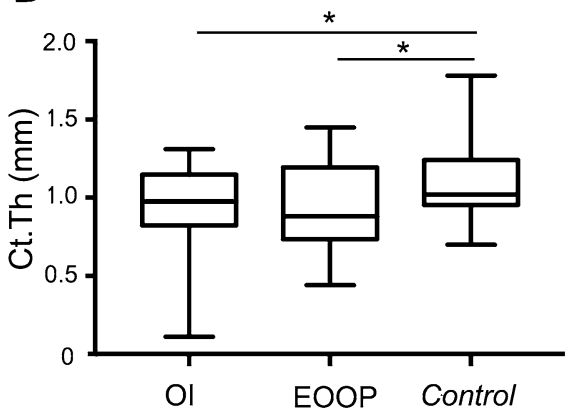

$\mathbf{F}$

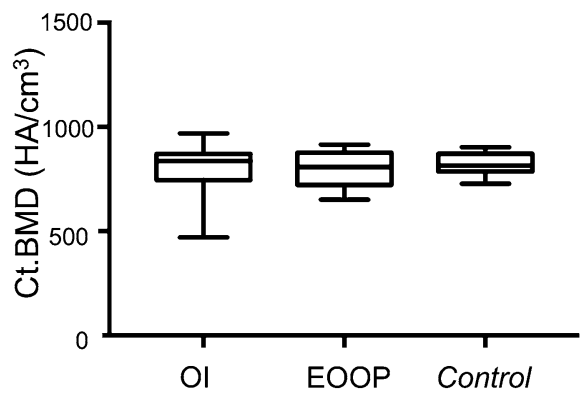

BMD but not cortical BMD were significantly lower in the distal tibia ( $p=0.011$; Fig. 4 ; Table 4$)$.

We next compared the OI patients with the EOOP cohort that was similar in age, DXA values, and fracture status (Table 2), but without any relevant mutation in 386 disease genes for skeletal disorders including collagen type I. This cohort demonstrated severe alterations in bone microstructure compared to healthy controls including a significant reduction in $\mathrm{BV} / \mathrm{TV}$ and $\mathrm{Tb} . \mathrm{N}$ in the radius and tibia as well as a decrease of Tb.Th in the radius and Ct.Th in the tibia (Figs. 3, 4; Tables 3, 4). When comparing the OI and EOOP cohort, $\mathrm{Ct}$. and $\mathrm{Tb}$. bone area as well as Ct.Pm were significantly lower in OI patients in the radius (Table 3). All other HR-pQCT parameters were essentially not different between OI and EOOP.

\section{Discussion}

In this study, we have demonstrated the in-depth skeletal phenotype of 29 adult OI patients with confirmed COL1AI or COL1A2 mutations and their microstructural similarities compared to a cohort of EOOP patients without clear disease-causing mutations. The genetic spectrum of OI is continuously growing [22], especially with the availability of modern techniques such as next-generation sequencing. Similar to previous reports [23, 24], the adult patients with mild OI included in this study showed a phenotypic and bone microstructural overlap with EOOP rather than the typical features of $\mathrm{OI}$ in most cases. We have recently also reported the detection of typical OI/collagen type I mutations in women with pregnancy-associated osteoporosis 
Table 4 Bone microstructure assessed by HR-pQCT (Tibia)

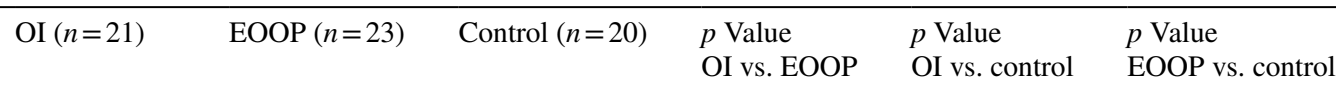

\begin{tabular}{|c|c|c|c|c|c|c|}
\hline \multicolumn{7}{|l|}{ Bone geometry } \\
\hline Ct. bone area $\left(\mathrm{mm}^{2}\right)$ & $96.56 \pm 7.69$ & $89.73 \pm 6.92$ & $127.7 \pm 7.63$ & 0.512 & 0.007 & 0.0007 \\
\hline Tb. bone area $\left(\mathrm{mm}^{2}\right)$ & $695.6 \pm 38.16$ & $651.4 \pm 63.64$ & $688.1 \pm 33.82$ & 0.569 & 0.883 & 0.629 \\
\hline \multicolumn{7}{|c|}{ Cortical and trabecular microstructure } \\
\hline Ct.Th (mm) & $0.875 \pm 0.071$ & $0.935 \pm 0.061$ & $1.122 \pm 0.063$ & 0.525 & 0.014 & 0.040 \\
\hline Ct.Pm (mm) & $112 \pm 2.83$ & $108 \pm 5.1$ & $114.1 \pm 2.53$ & 0.518 & 0.583 & 0.314 \\
\hline $\mathrm{BV} / \mathrm{TV}$ (ratio) & $0.095 \pm 0.008$ & $0.113 \pm 0.007$ & $0.162 \pm 0.01$ & 0.093 & $<0.0001$ & 0.0001 \\
\hline Tb.N (1/mm) & $1.428 \pm 0.098$ & $1.628 \pm 0.080$ & $2.143 \pm 0.089$ & 0.117 & $<0.0001$ & 0.0001 \\
\hline Tb.Th $(\mathrm{mm})$ & $0.066 \pm 0.004$ & $0.070 \pm 0.003$ & $0.075 \pm 0.003$ & 0.347 & 0.046 & 0.272 \\
\hline Tb.Sp (mm) & $0.727 \pm 0.095$ & $0.554 \pm 0.043$ & $0.409 \pm 0.023$ & 0.098 & 0.003 & 0.006 \\
\hline \multicolumn{7}{|c|}{ Volumetric bone mineral density } \\
\hline Total BMD (mg HA/ $\left./ \mathrm{cm}^{3}\right)$ & $219.9 \pm 16.29$ & $246.6 \pm 12.56$ & $306.3 \pm 13.98$ & 0.196 & 0.0003 & 0.003 \\
\hline Ct.BMD (mg HA $\left./ \mathrm{cm}^{3}\right)$ & $801.9 \pm 25.75$ & $797.9 \pm 18.03$ & $820.2 \pm 11.39$ & 0.898 & 0.519 & 0.319 \\
\hline Tb.BMD (mg HA/ $\left.\mathrm{cm}^{3}\right)$ & $130.9 \pm 20.65$ & $135.3 \pm 8.321$ & $194.4 \pm 11.44$ & 0.835 & 0.011 & 0.0001 \\
\hline
\end{tabular}

Bold indicates significant differences $(p<0.05)$

Data are presented as mean \pm SEM

[25]. Whether these patients with pathological mutations in COL1A1 or COL1A2 but inconsistent or missing clinical OI characteristics should be diagnosed as mild OI or as EOOP with collagen type I mutations is therefore still under discussion especially relating to the clinical picture.

While the evaluation of most variants through the different mutation databases and in combination with the clinical picture revealed pathogenic mutations, there were two borderline variants. Namely, the variant p.Pro205Ala in COL1A1 may not be causative but at least phenotype modifying; however, the phenotype was certainly typical for OI (Table 1). It has been associated with low bone mass [26] and was furthermore detected in patients with OI with an additional clear OI mutation [27]. The pathogenicity of the novel atypical missense mutation Asp1065Glu in COL1A1 was supported by segregation analysis; however, alternative splicing was not confirmed and the variant may thus be ranked as potentially pathogenic.

Regarding areal BMD measurements by DXA, $58.6 \%$ of the OI patients presented with low bone mass (i.e., DXA $Z$-score $<-2.0$ ). That OI patients may have a normal BMD has been shown before [28, 29], and the percentage of patients with osteoporosis according to DXA results was higher than expected in our adult cohort. After a decreasing fracture risk of OI patients during puberty and young adulthood, fracture risk typically rises in these patients during aging [1], which is due to the presence of additional risk factors for low bone mass and osteoporosis including age and menopause. Nonetheless, we could not detect an age-related decrease of DXA Z-scores in our adult OI patients.
In a previous histomorphometric study on iliac crest bone of OI patients, both cortical width and trabecular bone volume were clearly decreased in all OI types; and similarly to our findings the reduced trabecular bone mass was found to be largely due to a decreased trabecular number [30]. In fact, the microstructural HR-pQCT analysis revealed a predominant decrease in trabecular bone microstructure in both the OI and EOOP cohorts. In particular, we could detect a reduction in trabecular number in both cohorts. Compared to the control group, trabecular thickness at the distal radius was exclusively reduced in the EOOP cohort, while at the distal tibia it was exclusively reduced in the OI cohort. Interestingly, when comparing the OI and EOOP cohort, most HR-pQCT parameters besides bone geometry and cortical perimeter at the distal radius were found to be similar between the OI and EOOP cohort.

The biology of OI is different than postmenopausal or EOOP. This includes, i.e., changes in collagenous ECM as primary event as well as cellular stress leading to altered osteoblast function in OI [31, 32] vs. primary bone remodeling dysregulation in other bone loss conditions. However, a close monitoring is essential to not miss the appropriate time for a bone-specific therapy for adult OI patients, typically bisphosphonates, but also denosumab or teriparatide, as well as novel drugs like anti-sclerostin antibodies in the future [33]. Although we did not find an OI-specific microstructural pattern, HR-pQCT represents an additional tool to identify the patients with high risk of fracture that might not be identified by DXA [10]. The decision in favor or against a bone-specific therapy should be made with regard to presence of fractures, bone density, 
and microstructure as well as bone turnover markers. Further longitudinal studies are needed to assess the fracture risk and effectiveness of a bone-specific therapy in the aging OI population.

We are aware of the fact that OI is a very heterogeneous disorder especially regarding mutation types. Glycine substitution missense mutations lead to deposition of substantial quantities of abnormal collagen into the bone matrix. In contrast, nonsense mutations and frameshift mutations frequently lead to nonsense-mediated degradation, resulting in a quantitative deficit of predominantly normal collagen. Therefore, we have performed a subdivision in three groups based on the mutation types as opposed to previous studies that have compared bone microstructure in relation to Sillence OI type $[12,13]$. However, no clear differences in bone microarchitecture could be detected between the mutation groups.

Increases in bone turnover markers have been found previously in OI patients [34], which was confirmed in our cohort of adult patients. In fact, the number of patients with increased bone resorption markers would have been most likely even higher considering that nearly $50 \%$ had received a bone-specific therapy, mostly anti-resorptive, in the past or at present. In general, it has to be assumed that bone-specific treatments could have influenced the bone status (i.e., bone microstructure) in both the OI and EOOP cohorts.

In conclusion, we here point out that adult OI patients have a diminished trabecular bone structure, accompanied by reductions in trabecular number. It is noteworthy that these patients share many similarities in the microstructural parameters in comparison to EOOP patients without mutations in known disease genes. While no clear effects of OI mutation type on bone microstructure could be detected, it is reasonable to speculate on the different phenotypic effects of different COL1A1 and COL1A2 mutations as well as of other gene mutations on bone microstructure. However, further functional analyses of those mutations, for instance, in knock-in mouse models, are required to address this question.

Acknowledgements This project has received funding from the European Community's Seventh Framework Programme under Grant Agreement No. 602300 (SYBIL) and the German Federal Ministry of Education and Research (BMBF) within the project "Detection and Individualized Management of Early Onset Osteoporosis (DIMEOs)." Felix Schmidt acknowledges a PhD stipend of the Joachim Herz Stiftung in cooperation with the PIER initiative of the University of Hamburg and DESY Hamburg.

\section{Compliance with Ethical Standards}

Conflict of interest Tim Rolvien, Julian Stürznickel, Felix N. Schmidt, Sebastian Butscheidt, Tobias Schmidt, Björn Busse, Stefan Mundlos, Thorsten Schinke, Uwe Kornak, Michael Amling, and Ralf Oheim declare that they have no conflict of interest.
Human and Animal Rights and Informed Consent All procedures performed in studies involving human participants were in accordance with the ethical standards of the institutional and/or national research committee and with the 1964 Helsinki declaration and its later amendments or comparable ethical standards. Informed consent was obtained from all patients for the retrospective and anonymized database studies.

\section{References}

1. Forlino A, Marini JC (2016) Osteogenesis imperfecta. Lancet 387:1657-1671

2. Sillence DO, Senn A, Danks DM (1979) Genetic heterogeneity in osteogenesis imperfecta. J Med Genet 16:101-116

3. Forlino A, Cabral WA, Barnes AM, Marini JC (2011) New perspectives on osteogenesis imperfecta. Nat Rev Endocrinol 7:540-557

4. Keupp K, Beleggia F, Kayserili H, Barnes AM, Steiner M, Semler O, Fischer B, Yigit G, Janda CY, Becker J, Breer S, Altunoglu U, Grunhagen J, Krawitz P, Hecht J, Schinke T, Makareeva E, Lausch E, Cankaya T, Caparros-Martin JA, Lapunzina P, Temtamy S, Aglan M, Zabel B, Eysel P, Koerber F, Leikin S, Garcia KC, Netzer C, Schonau E, Ruiz-Perez VL, Mundlos S, Amling M, Kornak U, Marini J, Wollnik B (2013) Mutations in WNT1 cause different forms of bone fragility. Am J Hum Genet 92:565-574

5. Laine CM, Joeng KS, Campeau PM, Kiviranta R, Tarkkonen K, Grover M, Lu JT, Pekkinen M, Wessman M, Heino TJ, NieminenPihala V, Aronen M, Laine T, Kroger H, Cole WG, Lehesjoki AE, Nevarez L, Krakow D, Curry CJ, Cohn DH, Gibbs RA, Lee BH, Makitie O (2013) WNT1 mutations in early-onset osteoporosis and osteogenesis imperfecta. N Engl J Med 368:1809-1816

6. Saarinen A, Saukkonen T, Kivela T, Lahtinen U, Laine C, Somer M, Toiviainen-Salo S, Cole WG, Lehesjoki AE, Makitie O (2010) Low density lipoprotein receptor-related protein 5 (LRP5) mutations and osteoporosis, impaired glucose metabolism and hypercholesterolaemia. Clin Endocrinol 72:481-488

7. van Dijk FS, Zillikens MC, Micha D, Riessland M, Marcelis CL, de Die-Smulders CE, Milbradt J, Franken AA, Harsevoort AJ, Lichtenbelt KD, Pruijs HE, Rubio-Gozalbo ME, Zwertbroek R, Moutaouakil Y, Egthuijsen J, Hammerschmidt M, Bijman R, Semeins CM, Bakker AD, Everts V, Klein-Nulend J, CamposObando N, Hofman A, te Meerman GJ, Verkerk AJ, Uitterlinden AG, Maugeri A, Sistermans EA, Waisfisz Q, Meijers-Heijboer H, Wirth B, Simon ME, Pals G (2013) PLS3 mutations in X-linked osteoporosis with fractures. N Engl J Med 369:1529-1536

8. Gatti D, Colapietro F, Fracassi E, Sartori E, Antoniazzi F, Braga V, Rossini M, Adami S (2003) The volumetric bone density and cortical thickness in adult patients affected by osteogenesis imperfecta. J Clin Densitom 6:173-177

9. Boutroy S, Bouxsein ML, Munoz F, Delmas PD (2005) In vivo assessment of trabecular bone microarchitecture by high-resolution peripheral quantitative computed tomography. J Clin Endocrinol Metab 90:6508-6515

10. Sornay-Rendu E, Boutroy S, Munoz F, Delmas PD (2007) Alterations of cortical and trabecular architecture are associated with fractures in postmenopausal women, partially independent of decreased BMD measured by DXA: the OFELY study. J Bone Miner Res 22:425-433

11. Folkestad L, Hald JD, Hansen S, Gram J, Langdahl B, Abrahamsen B, Brixen K (2012) Bone geometry, density, and microarchitecture in the distal radius and tibia in adults with osteogenesis imperfecta type I assessed by high-resolution pQCT. J Bone Miner Res 27:1405-1412 
12. Kocijan R, Muschitz C, Haschka J, Hans D, Nia A, Geroldinger A, Ardelt M, Wakolbinger R, Resch H (2015) Bone structure assessed by HR-pQCT, TBS and DXL in adult patients with different types of osteogenesis imperfecta. Osteoporos Int 26:2431-2440

13. Hald JD, Folkestad L, Harslof T, Lund AM, Duno M, Jensen JB, Neghabat S, Brixen K, Langdahl B (2016) Skeletal phenotypes in adult patients with osteogenesis imperfecta-correlations with COL1A1/COL1A2 genotype and collagen structure. Osteoporos Int 27:3331-3341

14. Sykes B, Ogilvie D, Wordsworth P, Wallis G, Mathew C, Beighton P, Nicholls A, Pope FM, Thompson E, Tsipouras P, Schwartz R, Jensson O, Arnason A, Børresen A-L, Heiberg A, Frey D, Steinmann B (1990) Consistent linkage of dominantly inherited osteogenesis imperfecta to the type I collagen loci: COL1A1 and COL1A2. Am J Hum Genet 46:293-307

15. Mrosk J, Gandham SB, Shah H, Hecht J, Krüger U, Shukla A, Kornak U, Girisha KM (2018) Diagnostic strategies and genotype-phenotype correlation in a large Indian cohort of osteogenesis imperfecta. Bone 110:368-377

16. Rolvien T, Kornak U, Sturznickel J, Schinke T, Amling M, Mundlos S, Oheim R (2017) A novel COL1A2 C-propeptide cleavage site mutation causing high bone mass osteogenesis imperfecta with a regional distribution pattern. Osteoporos Int 29:243-246

17. Rolvien T, Koehne T, Kornak U, Lehmann W, Amling M, Schinke T, Oheim R (2017) A novel ANO5 mutation causing gnathodiaphyseal dysplasia with high bone turnover osteosclerosis. J Bone Miner Res 32:277-284

18. Zemojtel T, Kohler S, Mackenroth L, Jager M, Hecht J, Krawitz P, Graul-Neumann L, Doelken S, Ehmke N, Spielmann M, Oien NC, Schweiger MR, Kruger U, Frommer G, Fischer B, Kornak U, Flottmann R, Ardeshirdavani A, Moreau Y, Lewis SE, Haendel M, Smedley D, Horn D, Mundlos S, Robinson PN (2014) Effective diagnosis of genetic disease by computational phenotype analysis of the disease-associated genome. Sci Transl Med 6:252ra123

19. Milovanovic P, Adamu U, Simon MJ, Rolvien T, Djuric M, Amling M, Busse B (2015) Age- and sex-specific bone structure patterns portend bone fragility in radii and tibiae in relation to osteodensitometry: a high-resolution peripheral quantitative computed tomography study in 385 individuals. J Gerontol A 70:1269-1275

20. Mussawy H, Ferrari G, Schmidt FN, Schmidt T, Rolvien T, Hischke S, Ruther W, Amling M (2017) Changes in cortical microarchitecture are independent of areal bone mineral density in patients with fragility fractures. Injury 48:2461-2465

21. Schmidt T, Schmidt C, Schmidt FN, Butscheidt S, Mussawy H, Hubert J, Hawellek T, Oehler N, Barvencik F, Lohse AW, Schinke T, Schramm C, Amling M, Rolvien T (2018) Disease duration and stage influence bone microstructure in patients with primary biliary cholangitis. J Bone Miner Res 33(6):1011-1019

22. Pollitt R, McMahon R, Nunn J, Bamford R, Afifi A, Bishop N, Dalton A (2006) Mutation analysis of COL1A1 and COL1A2 in patients diagnosed with osteogenesis imperfecta type I-IV. Hum Mutat 27:716
23. Spotila LD, Constantinou CD, Sereda L, Ganguly A, Riggs BL, Prockop DJ (1991) Mutation in a gene for type I procollagen (COL1A2) in a woman with postmenopausal osteoporosis: evidence for phenotypic and genotypic overlap with mild osteogenesis imperfecta. Proc Natl Acad Sci USA 88:5423-5427

24. Styrkarsdottir U, Thorleifsson G, Eiriksdottir B, Gudjonsson SA, Ingvarsson T, Center JR, Nguyen TV, Eisman JA, Christiansen C, Thorsteinsdottir U, Sigurdsson G, Stefansson K (2016) Two rare mutations in the COL1A2 gene associate with low bone mineral density and fractures in Iceland. J Bone Miner Res 31:173-179

25. Butscheidt S, Delsmann A, Rolvien T, Barvencik F, Al-Bughaili M, Mundlos S, Schinke T, Amling M, Kornak U, Oheim R (2018) Mutational analysis uncovers monogenic bone disorders in women with pregnancy-associated osteoporosis: three novel mutations in LRP5, COL1A1, and COL1A2. Osteoporos Int. https://doi. org/10.1007/s00198-018-4499-4

26. Spotila LD, Colige A, Sereda L, Constantinou-Deltas CD, Whyte MP, Riggs BL, Shaker JL, Spector TD, Hume E, Olsen N et al (1994) Mutation analysis of coding sequences for type I procollagen in individuals with low bone density. J Bone Miner Res 9:923-932

27. Van Dijk FS, Nesbitt IM, Nikkels PG, Dalton A, Bongers EM, Van De Kamp JM, Hilhorst-Hofstee Y, Den Hollander NS, Lachmeijer AM, Marcelis CL (2009) CRTAP mutations in lethal and severe osteogenesis imperfecta: the importance of combining biochemi$\mathrm{cal}$ and molecular genetic analysis. Eur J Hum Genet 17:1560

28. Wekre LL, Eriksen EF, Falch JA (2011) Bone mass, bone markers and prevalence of fractures in adults with osteogenesis imperfecta. Arch Osteoporos 6:31-38

29. Paterson CR, Mole PA (1994) Bone density in osteogenesis imperfecta may well be normal. Postgrad Med J 70:104-107

30. Rauch F, Travers R, Parfitt AM, Glorieux FH (2000) Static and dynamic bone histomorphometry in children with osteogenesis imperfecta. Bone 26:581-589

31. Lisse TS, Thiele F, Fuchs H, Hans W, Przemeck GK, Abe K, Rathkolb B, Quintanilla-Martinez L, Hoelzlwimmer G, Helfrich M (2008) ER stress-mediated apoptosis in a new mouse model of osteogenesis imperfecta. PLoS Genet 4:e7

32. Mirigian LS, Makareeva E, Mertz EL, Omari S, Roberts-Pilgrim AM, Oestreich AK, Phillips CL, Leikin S (2016) Osteoblast malfunction caused by cell stress response to procollagen misfolding in $\alpha 2$ (I)-G610C mouse model of osteogenesis imperfecta. J Bone Miner Res 31:1608-1616

33. Glorieux FH, Devogelaer JP, Durigova M, Goemaere S, Hemsley S, Jakob F, Junker U, Ruckle J, Seefried L, Winkle PJ (2017) BPS804 anti-sclerostin antibody in adults with moderate osteogenesis imperfecta: results of a randomized phase 2a trial. J Bone Miner Res 32:1496-1504

34. Braga V, Gatti D, Rossini M, Colapietro F, Battaglia E, Viapiana O, Adami S (2004) Bone turnover markers in patients with osteogenesis imperfecta. Bone 34:1013-1016 